\title{
Safety and Efficacy of Oxcarbazepine: Results of Randomized, Double-Blind Trials
}

\begin{abstract}
Ahmad Beydoun, M.D.
Oxcarbazepine is approved as monotherapy and adjunctive therapy for partial seizures with and without secondarily generalized seizures in adults and as adjunctive therapy for partial-onset seizures in children aged 4-16 years. The clinical development of oxcarbazepine is different from the newer antiepileptic drugs (AEDs) in the extent and concordance of results across clinical trials. The safety and efficacy of oxcarbazepine was evaluated in adjunctive therapy trials, in comparative monotherapy trials with classic AEDs in adults and children with newly diagnosed epilepsy, in monotherapy therapeutic failure design trials in patients with refractory partial seizures, and in trigeminal neuralgia and affective disorder. The results of oxcarbazepine in treating epilepsy are discussed.
\end{abstract}

(Pharmacotherapy 2000;20(8 Pt 2):152S-158S)

Oxcarbazepine (10,11-dihydro-10-0x0-5H dibenz[b,f]azepine-5-carboxamide) is approved in 54 countries and recently was approved in the United States as monotherapy and adjunctive therapy for partial seizures with and without secondarily generalized seizures in adults and as adjunctive therapy for partial-onset seizures in children aged 4-16 years. Oxcarbazepine is the keto analog of carbamazepine (Figure 1). ${ }^{1}$ Although structurally related to carbamazepine, oxcarbazepine offers a number of clinically important pharmacokinetic advantages. One is its route of biotransformation. Unlike carbamazepine, which is metabolized by cytochrome P450 oxidative processes, oxcarbazepine undergoes primarily reductive biotransformation by cytosolic enzymes to the 10-monohydroxy metabolite, MHD. ${ }^{2}$ Although pharmacologically active, oxcarbazepine has a short half-life of only 1-2.5 hours versus a half-life of 8-10 hours for MHD, which is mainly responsible for the pharmacologic effect. ${ }^{3,4}$ At steady state, the plasma concentrations of MHD are 9-fold higher than those of the parent drug. ${ }^{3}$

\footnotetext{
From the University of Michigan Medical Center, Ann Arbor, Michigan.

Address reprint requests to Ahmad Beydoun, M.D., University of Michigan Medical Center, University Hospital 1B300/0036, 1500 East Medical Center Drive, Ann Arbor, MI 48109; e-mail: Beydoun@umich.edu.
}

The lack of oxidative metabolism results in two attractive properties. First, the 10,11 epoxide that contributes to the adverse event profile of carbamazepine $e^{5,6}$ is not produced. In addition, oxcarbazepine has a lower propensity to inhibit or induce hepatic oxidative enzymes and therefore a diminished potential for drug-drug interactions. $^{2}$ Also, whereas carbamazepine induces its own metabolism and undergoes autoinduction, the elimination of oxcarbazepine and its metabolites does not change significantly over time. ${ }^{3}$ Similar to carbamazepine, the anticonvulsant properties of oxcarbazepine probably are mediated in part by blockage of the voltage-dependent sodium channel. ${ }^{1,2}$

The clinical development of oxcarbazepine is different from the newer antiepileptic drugs (AEDs) in the extent and concordance of clinical trial results. The results obtained from clinical trials with oxcarbazepine for the treatment of epilepsy are discussed. All trials were approved by the institutional review boards of the participating centers, and all patients and legal guardians gave informed consent.

\section{Adjunctive Therapy Trials}

Two double-blind, placebo-controlled trials evaluated the efficacy and safety of oxcarbazepine as adjunctive therapy. ${ }^{7,8}$ The first was a 


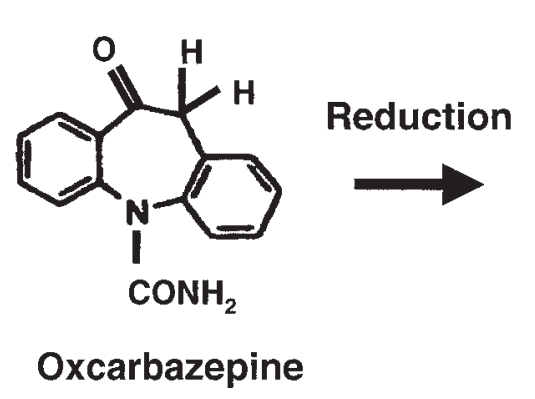<smiles>NC(=O)N1C=CCc2ccccc21</smiles>

Carbamazepine

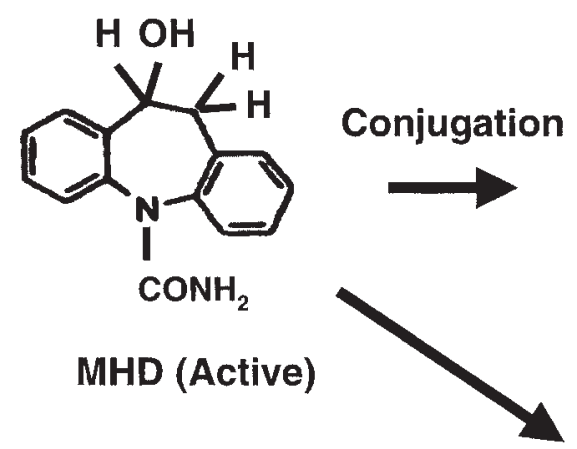<smiles>NC(=O)N1C=CC2CC2(O)c2ccccc21</smiles>

10, 11-Epoxide (Active)<smiles>NC(=O)N1c2ccccc2C(C(=O)O)C(C(=O)O)(C(=O)O)c2ccccc21</smiles>

\section{Glucuronide \\ Conjugate}

Autoinduction

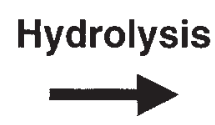

Figure 1. Metabolic pathway of oxcarbazepine and carbamazepine. Reprinted with permission from reference 1.

multicenter, randomized, four-arm, parallelgroup trial with oxcarbazepine at daily doses of $600 \mathrm{mg}, 1200 \mathrm{mg}$, and $2400 \mathrm{mg}$ in patients with medically refractory partial epilepsy. ${ }^{7}$ Eligible patients were 15-65 years of age, with at least four seizures/month during the baseline phase. The primary efficacy variable was the percentage change in seizure frequency/28 days in the double-blind treatment phase relative to the baseline phase.

A total of 173 patients were treated in the placebo group and 519 in the oxcarbazepine groups- 168 receiving $600 \mathrm{mg} /$ day, 177 receiving $1200 \mathrm{mg} /$ day, and 174 receiving $2400 \mathrm{mg} /$ day. The results of the primary efficacy analysis demonstrated a dose-response relationship, with a median reduction in seizure frequency for placebo, oxcarbazepine $600 \mathrm{mg} /$ day, $1200 \mathrm{mg} / \mathrm{day}$, and $2400 \mathrm{mg} /$ day of $8 \%, 26 \%, 40 \%$ and $50 \%$, respectively. All oxcarbazepine-treated patients showed significant improvement compared with placebo-treated patients ( $p=0.0001$ ).

The most common adverse events were related to the central nervous system (dizziness, headache, somnolence, ataxia, nystagmus, abnormal gait) and digestive system (vomiting, nausea, abdominal pain). Some adverse events appeared to be dose related: dizziness, diplopia, somnolence, vomiting, nausea, ataxia, nystagmus, abnormal vision, vertigo and abnormal gait. Overall, no clinically noteworthy trends were observed in vital signs or laboratory values. The results support the efficacy of oxcarbazepine as adjunctive therapy in patients with refractory partial seizures, which include the seizure subtypes of simple, complex, and partial seizures evolving to secondarily generalized tonic-clonic seizures.

The second adjunctive therapy trial evaluated the effectiveness of oxcarbazepine in children with inadequately controlled partial seizures. ${ }^{8} \mathrm{~A}$ total of 267 children aged 3-17 years, maintained on one or two AEDs, were randomized to a placebo-controlled, double-blind treatment phase (consisting of a 14-day titration period followed by a 98-day maintenance period) after meeting the treatment phase entry criteria of at least eight partial seizures during the 56-day baseline phase. The intent-to-treat efficacy analysis included 264 of 267 patients; 3 patients (2 oxcarbazepine, 1 placebo) discontinued prematurely with no seizure information available. A flexible titration schedule was used; dosages could be adjusted to allow individual patients to reach a maximum tolerated dosage. The target randomized dosage for the appropriate weight category (30-46 $\mathrm{mg} / \mathrm{kg} /$ day) was not exceeded. Efficacy variables were the same in both trials. 
Table 1. Oxcarbazepine Comparative Monotherapy Trials

\begin{tabular}{lcclc}
\hline Comparator & Number & Age $($ yrs $)$ & Seizure type & Duration (wks) \\
\hline Phenytoin $^{9}$ & 287 & $16-65$ & Partial/GTC & 56 \\
Phenytoin $^{10}$ & 193 & $5-18$ & Partial/GTC & 56 \\
Val proate $^{11}$ & 249 & $15-65$ & Partial/GTC & 56 \\
Carbamazepine $^{12}$ & 235 & $15-65$ & Partial/GTC & 56 \\
\hline
\end{tabular}

Partial/GTC = partial seizures with or without secondarily generalized seizures and generalized tonicclonic seizures without partial onset.

As demonstrated with adults, oxcarbazepine was found to be an effective adjunctive treatment in children. The median partial-seizure frequency decreased from baseline by $35 \%$ in patients treated with oxcarbazepine compared with a median reduction of $9 \%$ in patients receiving placebo $(p=0.0001)$. Premature discontinuations due to adverse events were $10 \%$ (14/138) in the oxcarbazepine group and 3\% (4/129) in the placebo group. The most common adverse events resulting in premature discontinuation were vomiting and nausea. O verall, no clinically noteworthy trends were observed in vital signs or laboratory values.

\section{Comparative Monotherapy Trials}

The safety and efficacy of oxcarbazepine monotherapy was evaluated in four international trials in patients with newly diagnosed partial seizures or generalized tonic-clonic seizures (Table 1). ${ }^{9-12}$ All four trials were multicenter, double-blind, parallel-group design that randomized patients to treatment with oxcarbazepine or one of the standard AEDs.

Eligible patients had to have a minimum of two seizures separated by at least 8 hours within 6 months preceding trial entry. No previous AED use was allowed, except for emergency treatment of seizures for a maximum of 3 weeks prior to trial entry. The trials consisted of an 8-week titration phase followed by a 48-week maintenance period. The primary efficacy variable was the proportion of seizure-free patients during the maintenance phase. Secondary outcome parameters consisted of the proportion of patients who discontinued because of adverse experiences and the time to premature discontinuation due to adverse events.

Oxcarbazepine was compared with phenytoin in two trials. ${ }^{9,} 10$ In the adult trial, 287 patients (aged 16-65 yrs) were enrolled, ${ }^{9}$ and in the pediatric trial, 193 patients (aged 5-18 yrs) were enrolled. ${ }^{10}$ After 8 weeks, patients were placed on a dosing schedule of 3 times/day, with daily doses of 150-800 mg for phenytoin and 450-2400 mg for oxcarbazepine. The therapeutic range was defined as $10-25 \mathrm{mg} / \mathrm{L}$ for phenytoin and 15-35 mg/L for MHD, although no therapeutic range for MHD was identified consistently. The mean daily dose of oxcarbazepine at the start of the maintenance phase was 1028 $\mathrm{mg}$ in adults (range 600-2100 mg) and $672 \mathrm{mg}$ in children and adolescents (range 300-1350 $\mathrm{mg}$ ). The corresponding average daily phenytoin dose was $313 \mathrm{mg}$ in adults (range 100-650 mg) and $226 \mathrm{mg}$ in children and adolescents (range 100-400 mg).

In both trials, phenytoin and oxcarbazepine had similar efficacy, with $58-60 \%$ of patients remaining seizure free during the maintenance phase. However, oxcarbazepine was tolerated significantly better than phenytoin in both trials, with time to premature discontinuation due to adverse events significantly shorter for phenytoin compared with oxcarbazepine. The withdrawal rates due to adverse events were 2-4\% for patients treated with oxcarbazepine and $11-15 \%$ for patients randomized to phenytoin.

In the comparative trial of oxcarbazepine versus valproate, 249 patients (aged 15-65 yrs) were randomized. ${ }^{11}$ After an 8-week baseline phase, patients were placed on a dosing schedule of 3 times/day, with daily doses of 900-2400 mg for valproate or oxcarbazepine. The average daily dose of oxcarbazepine at the start of the maintenance phase was 1053 mg (range 600-2400 mg) and of valproate was $1146 \mathrm{mg}$ (range 600-2700 mg).

Results showed no significant differences in seizure-free patients or tolerability between treatment groups. In this trial, $57 \%$ of patients randomized to oxcarbazepine remained seizure free during the maintenance phase compared with $54 \%$ of valproate patients. Analysis of time to premature discontinuation due to adverse events was not significantly different between the two groups. The withdrawal rates due to adverse events were $12 \%$ and $8 \%$ in the oxcarbazepine 
and val proate groups, respectively.

The fourth trial compared oxcarbazepine versus carbamazepine in 235 patients aged 15-65 yrs. ${ }^{12}$ The duration of the titration phase was 4-8 weeks, followed by a maintenance phase of up to 48 weeks. The mean final daily dose of oxcarbazepine was $1040 \mathrm{mg}$ (range 300-1800 $\mathrm{mg}$ ) and of carbamazepine was $684 \mathrm{mg}$ (range 300-1400 mg).

No significant difference in efficacy was observed between the two groups, with $52 \%$ and $60 \%$ of oxcarbazepine and carbamazepine patients, respectively, remaining seizure free throughout the maintenance phase. However, oxcarbazepine was tolerated significantly better. Withdrawal rates due to significant adverse events were $14 \%$ with oxcarbazepine and $26 \%$ with carbamazepine. Of interest, 16 patients discontinued carbamazepine because of cutaneous rashes compared with 9 patients on oxcarbazepine.

\section{Monotherapy Therapeutic Failure Design Trials}

An additional four multicenter therapeutic failure design trials were conducted to demonstrate the safety and efficacy of oxcarbazepine as monotherapy. ${ }^{13-16}$ Two trials were double-blind, placebo-controlled, ${ }^{13,}{ }^{14}$ and two were double-blind, dose-controlled, monotherapy conversion trials using high-dose ( $2400 \mathrm{mg} /$ day) versus low-dose ( $300 \mathrm{mg} /$ day) oxcarbazepine as a substitute for one or more AEDs. ${ }^{15,16}$

Placebo-Controlled Trial in Patients with RecentOnset, Partial-Onset Seizures

The objective of this randomized, parallelgroup trial was to evaluate the safety and efficacy of oxcarbazepine monotherapy in untreated patients aged 8-69 years with newly or recently diagnosed partial-onset seizures. ${ }^{13}$

After completing a 56-day baseline phase, which could be retrospective, eligible patients with at least two seizures/month were randomized to placebo or oxcarbazepine 1200 $\mathrm{mg} /$ day (starting at $300 \mathrm{mg}$ twice/day and titrated over 6 days to $1200 \mathrm{mg} /$ day, given as $600 \mathrm{mg}$ twice/day) and then maintained in the doubleblind treatment phase for 84 days. A total of 67 patients were randomized, 32 to oxcarbazepine and 35 to placebo. The primary efficacy variable- time to first partial seizure-was significantly longer in the oxcarbazepine group. The median time to first partial seizure was approximately 12 days in the oxcarbazepine group compared with approximately 3 days in the placebo group. The reduction in seizure frequency compared with baseline also was significantly better with oxcarbazepine, with a median reduction of $89 \%$ and $37 \%$ in the oxcarbazepine and placebo groups, respectively.

During the double-blind treatment phase, the percentage of patients who reported at least one adverse event, whether or not related to study drug, was $78 \%$ for the oxcarbazepine group and $86 \%$ for the placebo group. Increased appetite, vomiting, and paresthesias were reported more often in the placebo group, whereas dizziness and upper respiratory infection were more frequent in the oxcarbazepine group. Three patients in the oxcarbazepine group and two in the placebo group withdrew from the trial because of adverse events. No patient withdrew because of a laboratory abnormality, and no serious adverse events occurred.

Inpatient Monotherapy Trial in Patients Undergoing Evaluation for Epilepsy Surgery

The efficacy and safety of oxcarbazepine monotherapy was evaluated in a multicenter, double-blind, randomized, placebo-controlled, two-arm, parallel-group inpatient trial in patients aged 11-65 years undergoing evaluation for epilepsy surgery. ${ }^{14}$ After completion of their presurgical diagnostic evaluation, patients who had their AEDs discontinued and who satisfied the inclusion and exclusion criteria were randomized to treatment with oxcarbazepine $1200 \mathrm{mg}$ twice daily ( $1500 \mathrm{mg} /$ day on day 1 and $2400 \mathrm{mg} /$ day thereafter) or placebo. Patients remained in the trial for 10 days or until they experienced one of the following study-defined exit criteria: a total of four seizures, two newonset secondarily generalized seizures, serial seizures, or status epilepticus.

A total of 102 patients, 51 in each treatment arm, were randomized. The primary efficacy variable-time to meeting one of the exit criteria-was significantly longer in oxcarbazepine patients compared with placebo. A worst-case scenario-where oxcarbazepine patients who dropped out were classified as having exited the trial and placebo patients who dropped out were classified as having completed the trial-was still highly significantly in favor of the oxcarbazepine group. In this scenario, $47 \%$ of oxcarbazepine patients would have met an exit criterion compared with $84 \%$ of placebo patients 
$(p<0.0001)$.

Twenty-five percent of oxcarbazepine patients remained seizure free throughout the trial compared with $2 \%$ of those randomized to placebo. If seizure freedom were assessed beginning at day 2 (the first day of oxcarbazepine at $2400 \mathrm{mg} / \mathrm{day}$ ), 31\% of oxcarbazepine patients were seizure free compared with $4 \%$ of placebo patients. The secondary outcome variables were concordant and favored the oxcarbazepine group. Oxcarbazepine patients had a median seizure frequency/9 days of 2 seizures compared with a median frequency of 31 seizures for placebo patients $(p=0.0001)$. In this trial, $8 \%$ of oxcarbazepine patients compared with $47 \%$ of placebo patients experienced secondarily generalized seizures $(p=0.0006)$.

The adverse experiences observed most frequently and considered trial-drug related were dizziness, nausea, somnolence, headache, diplopia, fatigue, and vomiting. The majority of these were rated as mild or moderate in severity. Two oxcarbazepine patients were discontinued from the trial due to adverse experiences. One developed a rash, and another patient with a history of psychosis experienced a postictal psychotic episode. There were no clinically noteworthy abnormal findings among oxcarbazepine patients in physical examinations, vital signs, or clinical laboratory parameters.

Dose-Controlled Monotherapy Trials in Outpatients with Refractory Partial Epilepsy

Two dose-controlled monotherapy trials compared oxcarbazepine $2400 \mathrm{mg} /$ day with oxcarbazepine $300 \mathrm{mg} /$ day. ${ }^{15,16}$ The first was a multicenter, randomized, double-blind, parallelgroup trial that compared the safety and efficacy of oxcarbazepine monotherapy in patients aged 12 years and older with medically refractory partial seizures. ${ }^{15}$ Patients who experienced 2-40 partial-onset seizures/28 days during a 56-day baseline phase while maintained on constant doses of one or two AEDs were randomized to one of the two oxcarbazepine groups.

The 126-day double-blind phase comprised a 14-day titration period and a 112-day maintenance period. Patients completed the trial by either completing the double-blind phase or meeting one of four exit criteria: 2-fold increase in partial-seizure frequency compared with baseline in a 28-day period; 2 -fold increase in the highest consecutive 2-day partial-seizure frequency; occurrence of a single generalized seizure, if none occurred during the 6 months prior to randomization; or prolongation or worsening of generalized seizure duration or frequency requiring intervention.

A total of 87 patients were randomized, 41 in the $2400-\mathrm{mg} / \mathrm{day}$ group and 46 in the $300-$ $\mathrm{mg} /$ day group. The percentage of patients meeting one of the exit criteria (the primary efficacy variable) was significantly lower $(p<0.0001)$ for the $2400-\mathrm{mg} /$ day group $(41 \%)$ compared with the 300-mg/day group (93\%). In the intent-to-treat analysis for the oxcarbazepine $2400-\mathrm{mg} /$ day group, $42 \%$ of patients had at least a $50 \%$ reduction in seizures and $12 \%$ were seizure free compared with $7 \%$ and $0 \%$, respectively, for the 300-mg/day group.

Adverse experiences that were more common in the 2400-mg/day group were dizziness, fatigue, headache, somnolence, nausea, and vomiting. The most common treatment-limiting adverse experiences involved the central nervous system (dizziness, headache, somnolence) and digestive system (nausea, vomiting). In this trial, premature discontinuation rates due to adverse events were $2.2 \%(1 / 46)$ in the $300-\mathrm{mg} /$ day group and $14.6 \%$ $(6 / 41)$ in the $2400-\mathrm{mg} /$ day group. One patient withdrew due to hyponatremia (baseline sodium $137 \mathrm{mEq} / \mathrm{L}$; day 10 sodium $121 \mathrm{mEq} / \mathrm{L}$ ), with discontinuation of oxcarbazepine resulting in normalization of sodium levels within 4 days. All withdrawals due to adverse events occurred during the conversion phase, when patients received oxcarbazepine concomitantly with their baseline AEDs.

The second dose-controlled trial was conducted in patients whose partial seizures were inadequately controlled while maintained on carbamazepine monotherapy at a daily dose of 800-1600 mg. ${ }^{16}$ Entry criteria included the occurrence of 2-40 partial seizures during a 28day screening phase. The trial consisted of five phases: a 56-day screening phase (patients maintained on carbamazepine monotherapy); a 28-day, open-label conversion phase (patients tapered off carbamazepine by $25 \%$ per week, with oxcarbazepine simultaneously titrated in $600-\mathrm{mg}$ increments weekly over 3 weeks to 2400 $\mathrm{mg} /$ day); a 56-day, open-label baseline phase (patients maintained on oxcarbazepine 2400 $\mathrm{mg} /$ day); and a 126-day, double-blind treatment phase (patients randomized to continue the oxcarbazepine dosage at $2400 \mathrm{mg} / \mathrm{day}$ or to be tapered to a daily dose of $300 \mathrm{mg}$ ).

The exit criteria were similar to those of the previously described dose-response trial, with the 
seizure frequency during a double-blind phase being compared with that during the 56-day, open-label baseline phase. The primary efficacy variable was time to meeting one of the exit criteria. In addition, the percentage of patients meeting one of the exit criteria in each treatment group was compared.

$\mathrm{N}$ inety-six patients were randomized to the double-blind treatment phase, with 94 patients aged 12-65 years included in the efficacy analysis. Time to meeting one of the exit criteria was significantly longer for patients treated with $2400 \mathrm{mg} /$ day compared with those randomized to $300 \mathrm{mg} /$ day. No patients were discontinued prematurely due to adverse events. Adverse events that were more common in the oxcarbazepine $2400-\mathrm{mg} /$ day group were fatigue, nausea, ataxia, and headache. In addition, no clinically noteworthy trends were observed in vital signs or laboratory values.

\section{Use of Oxcarbazepine in Clinical Practice}

As demonstrated in the comparative clinical trials, oxcarbazepine has equal efficacy to the standard AEDs as monotherapy and is significantly better tolerated than phenytoin and carbamazepine. This makes it an attractive alternative first-line agent for the treatment of patients with newly diagnosed partial-onset or generalized tonic-clonic seizures. In addition, it has shown definitive evidence of efficacy as monotherapy in the dose-response and placebocontrolled therapeutic failure design trials.

Compared with carbamazepine, oxcarbazepine has similar efficacy with more attractive pharmacokinetic and safety profiles. It does not undergo autoinduction, has fewer propensities for heteroinduction, and is associated with less frequent rashes. Patients who have had a hypersensitivity reaction to carbamazepine should be informed that $25-30 \%$ of them will experience hypersensitivity to oxcarbazepine. ${ }^{2}$ There were no significant interactions when oxcarbazepine was administered concomitantly with warfarin, ${ }^{17}$ verapamil, ${ }^{2,18}$ dextropropoxyphene, ${ }^{19}$ cimetidine, ${ }^{20}$ or erythromycin. ${ }^{21}$ Although oxcarbazepine is associated with significantly less drug-drug interactions compared with carbamazepine, it will induce the metabolism of oral contraceptives substantially and could result in breakthrough bleeding. ${ }^{22}$ The teratogenic effect of oxcarbazepine in humans has not yet been characterized adequately. In addition to folic acid supplementation, the same precautions that apply to other AEDS also apply to oxcarbazepine.

Clinical trials have shown that the effective dose of oxcarbazepine is approximately $50 \%$ higher than for carbamazepine. When oxcarbazepine is substituted for carbamazepine, replacement of every $200 \mathrm{mg}$ of carbamazepine with $300 \mathrm{mg}$ oxcarbazepine is suggested. The substitution can be gradual over a period of days or it can be done at once, as in the case of an allergic reaction to carbamazepine. ${ }^{23}$ Replacement of carbamazepine with oxcarbazepine will result in deinduction of the liver cytochrome P450 enzyme system function. ${ }^{24,25}$ This could be clinically relevant for patients receiving concomitant valproate or phenytoin therapy because serum levels might increase by up to $20 \%$. This deinduction also can be used clinically when polytherapy with valproate and carbamazepine is considered. In such cases, it is difficult to achieve adequate valproate serum levels because of the induction caused by carbamazepine. Replacing carbamazepine with oxcarbazepine should circumvent this problem.

Oxcarbazepine can be initiated at a clinically effective dosage of $300 \mathrm{mg}$ twice/day. However, clinical experience suggests that a starting dosage of $150 \mathrm{mg}$ twice/day is much better tolerated. The dosage can be increased in 300-600-mg increments. Typically, the effective daily dose range for patients with newly diagnosed epilepsy is 600-1200 mg; for patients with medically refractory partial epilepsy, daily doses up to 2400 $\mathrm{mg}$ may be needed. Dosage adjustment of oxcarbazepine is recommended for patients with creatinine clearances bel ow $30 \mathrm{ml} /$ minute.

\section{Conclusion}

Oxcarbazepine has accumulated more than 200,000 patient-years of exposure. Overall, the results of clinical trials demonstrate that oxcarbazepine is an important option for the initiation of monotherapy and for adjunctive use in the treatment of partial seizures, with excellent tolerability and reduced risk of drug interactions when compared with the standard AEDs.

\section{References}

1. Schacter SC. Oxcarbazepine: current status and clinical applications. Exp Opin Invest Drugs 1999;8:1-10.

2. Novartis Pharmaceuticals Corporation. Trileptal (oxcarbazepine) package insert. East Hanover, NJ;2000.

3. Dickinson RG, Hooper W D, Dunstan PR, Eadie MJ. First dose and steady-state pharmacokinetics of oxcarbazepine and its 10hydroxy metabolite. Eur J Clin Pharmacol 1989;37:69-74.

4. Lloyd P, Flesch G, Dieterle W. Clinical pharmacology and pharmacokinetics of oxcarbazepine. Epilepsia 1994;35:S10-3. 
5. Patsalos PN, Stephenson TJ, Krishna S, Elyas AA, Lascelles PT, Wiles CM. Side-effects induced by carbamazepine-10,11epoxide [letter]. Lancet 1985;2:1432.

6. Wamil AW, Schmutz M, Portet C, Feldmann KF, McLean MJ. Effects of oxcarbazepine and 10-hydroxycarbamazepine on action potential firing and generalized seizures. Eur J Pharmacol 1994;271:301-8.

7. Barcs G, Walker EB, Elger CE, et al. Oxcarbazepine placebocontrolled, dose-ranging trial in refractory partial epilepsy. Epilepsia, in press.

8. Glauser TA, Nigro M, Sachdeo R, et al. Adjunctive therapy with oxcarbazepine in children with partial seizures. Neurology 2000;54:2237-44.

9. Bill PA, Vigonius $\mathbf{U}$, Pohlmann $\mathbf{H}$, et al. A double-blind controlled clinical trial of oxcarbazepine versus phenytoin in adults with previously untreated epilepsy. Epilepsy Res 1997;27:195-204.

10. Guerreiro MM, Vigonius $\mathbf{U}$, Pohlmann $\mathbf{H}$, et al. A double-blind controlled clinical trial of oxcarbazepine versus phenytoin in children and adolescents with epilepsy. Epilepsy Res 1997;27:205-13.

11. Christe W, Kramer G, Vigonius U, et al. A double-blind controlled clinical trial: oxcarbazepine versus sodium valproate in adults with newly diagnosed epilepsy. Epilepsy Res 1997:26:451-60.

12. Dam M, Ekberg R, Loyning Y, Waltimo 0, Jakobsen K. A double-blind study comparing oxcarbazepine and carbamazepine in patients with newly diagnosed, previously untreated epilepsy. Epilepsy Res. 1989;3:70-6.

13. Sachdeo RC, Edwards K, Hasegawa $\mathbf{H}$, et al. Safety and efficacy of oxcarbazepine $1200 \mathrm{mg} /$ day in patients with recentonset partial epilepsy [abstr]. N eurology 1999;52(suppl 2):A391.

14. Schachter SC, Vazquez B, Fisher RS, et al. Oxcarbazepine: double-blind, randomized, placebo-control, monotherapy trial for partial seizures. Neurology 1999;52:732-7.
15. Beydoun A, Sachdeo RC, Rosenfeld WE, et al. Oxcarbazepine monotherapy for partial-onset seizures: a multicenter, doubleblind, clinical trial. Neurology 2000;54:2245-51.

16. Sachdeo R, Beydoun A, Schachter S, et al. Safety and efficacy of oxcarbazepine monotherapy [abstr]. Neurology 1998;50:A200.

17. Kramer G, Tettenborn B, Jensen PK, Menge GP, Stoll KD Oxcarbazepine does not affect the anticoagulant activity of warfarin. Epilepsia 1992;33:1145-8.

18. Kramer G, Tettenborn B, Flesch G. Oxcarbazepine-verapamil drug interaction in healthy volunteers. Epilepsia 1991;32 (suppl 1):70-1.

19. Mogensen PH, Jorgensen L, Boas J, et al. Effects of dextropropoxyphene on the steady-state kinetics of oxcarbazepine and its metabolites. Acta Neurol Scand 1992;85:14-7.

20. Keranen T, Jolkkonen J, Klosterskov-Jensen P, Menge G P Oxcarbazepine does not interact with cimetidine in healthy volunteers. Acta N eurol Scand 1992;85:239-42.

21. Keranen $\mathbf{T}$, Jolkkonen J, Jensen PK, Menge GP, Andersson $\mathbf{P}$. Absence of interaction between oxcarbazepine and erythromycin. Acta N eurol Scand 1992;86:120-3.

22. Klosterskov Jensen P, Saano V, Haring P, Svenstrup B, Menge GP. Possible interaction between oxcarbazepine and an oral contraceptive. Epilepsia 1992;33:1149-52.

23. Reinikainen $\mathbf{K J}$, Keranen $\mathrm{T}$, Halonen $\mathrm{T}$, Komulainen $\mathbf{H}$, Riekkinen PJ. Comparison of oxcarbazepine and carbamazepine: a double-blind study. Epilepsy Res 1987;1:284-9.

24. Isojarvi JI, Pakarinen AJ, Rautio A, Pelkonen 0, Myllyla VV Liver enzyme induction and serum lipid levels after replacement of carbamazepine with oxcarbazepine. Epilepsia 1994;35:1217-20.

25. Faigle JW, Menge GP. Metabolic characteristics of oxcarbazepine (Trileptal ${ }^{\circledR}$ ) and their beneficial implications for enzyme induction and drug interactions. Behav Neurol 1990;3(suppl):S21-30. 